\title{
Disposable spectrofluidic devices for attenuated total reflection infrared spectroscopy: characterization sensitivity, spatial resolution and generally applicable to multiple device types.
}

Nan Jia, ${ }^{a}$ Arthur Daignault-Bouchard, ${ }^{\mathrm{b}}$ Tianyang Deng, ${ }^{a}$ Leon Torres de Oliveira, ${ }^{a}$ André BéginDrolet $^{\mathrm{b}}$ and Jesse Greener ${ }^{\mathrm{a}, \mathrm{c}}$

a Département de chimie, Faculté des sciences et de génie, Université Laval, Québec, QC G1V 0A6, Canada

${ }^{\text {b }}$ Département de génie mécanique, Faculté des sciences et de génie, Université Laval, Québec, QC G1V 0A6, Canada

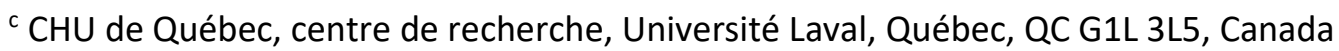

Keywords: Spectrofluidic, microfluidic, attenuated total reflection, Fourier transform infrared spectroscopy, assays, laminar co-flow, microreactors

\section{Abstract}

In this paper, we present a generalizable method for the fabrication of disposable spectrofluidic devices for solution characterization by attenuated total reflectance (ATR) Fourier transform infrared (FTIR) spectroscopy. A major design feature is the integration of an ATR element into the device rather than the fabrication of the microchannels on its sensing surface. This alleviates spatial limitations, due to small element footprint and dominance of edge-beading, enabling arbitrarily complex microfluidic circuitry and complex world-to-chip interfaces while leaving the entire ATR element available for sensing. An optimized optical interface maximizes light transfer 
into the on-chip sensing chamber. This promotes low limits of detection, fast measurements and/or designs featuring multiple sensing sub regions. To demonstrate the approach, we conducted measurements on complex flow profiles generated from four separate proof-ofconcept spectrofluidic devices. A high sensitivity device detected glucose and sodium phosphate dibasic $\left(\mathrm{Na}_{2} \mathrm{HPO}_{4}\right)$ at concentrations as low as $3 \mathrm{mM}$ and $1 \mathrm{mM}$, respectively or and for time-lapse results with second-scale time resolution from single-scan measurements. We also demonstrated spatial selectivity for assays in parallel channels, measurements of concentration gradients in a multi-laminar co-flow device, and monitored fast kinetics of the protonation of a pH buffer in a microfluidic reactor.

\section{Introduction:}

For several decades, different aspects of microfluidic technology have been developed in parallel in academic and private sector, with over of focus being new fabrication techniques, device designs, fluid control systems, world-to-chip interfaces and on-chip characterization. The last is arguably the most difficult since it usually involves challenging device fabrication protocol to embed probes and coupling to peripheral equipment. This is especially true for infrared (IR) spectroscopy because, other than for a few notable exceptions, ${ }^{1}$ measurements in microchannels are usually carried out in attenuated total reflection (ATR) mode, which avoids signal attenuation by the device and analyte fluids. For examples, reversibly integrated ATR probes have been demonstrated for single point measurement leading coupling of probe light focused into to a small fixed detection zone along the flow path. ${ }^{2}$ The approach was successfully used to measure the kinetics of a complicated polymerization reaction by modulating reaction time by changes to flow rates, ${ }^{3}$ and was also compatible with other on-chip probes, ${ }^{4}$ but spatial-resolved measurements 
were not possible. Moreover, the ATR coupling to the device required highly engineered approaches and could result in dead volumes and leaks if not done accurately. Another published approach for on-chip FTIR is to fabricate the microfluidic channel structure on top of the ATR crystal. Previously we devised an approach to seal PDMS channels with a large monolithic germanium ATR crystal surface. The entire assembly could be translated mechanically to interrogate different locations on-chip. ${ }^{5}$ The system featured either 6 parallel channels, for channel-specific assay measurements, or a single channel device that could be translated to create one-dimensional maps along the channel length. The setup was complemented with inline reflection microscopy to monitor the formation of bacterial biofilms. Another approach to chemically image the contents of a microfluidic device with high spatial resolution in two dimensions was accomplished using a $64 \times 64$ pixel focal plane array (FPA) detector to sub-divide the radiation leaving a large single-bounce ATR crystal that was adhered to the bottom side of a microfluidic device. ${ }^{6}$ Also using a FPA, a simple Y-shape microfluidic was recently imaged using a low-cost multi-ridge silicon ATR crystal. And what is more, by changing the focal length of the last off-axis parabolic mirror with a lens, the resolution could nearly reach the diffraction limit. ${ }^{7}$ Such multi-grove ATR crystals distinguish themselves among other methods for integrated reflection elements for IR characterization in microscale flow chemistry devices ${ }^{8}$ due to their ability to meet central goals of low-cost, disposable microfluidic devices without any sacrifice to sensitivity. To further reduce the system cost, our group demonstrated a method to replace the FPA with a standard single point detector. ${ }^{9}$ The detector recorded separate spectra based on the position of an automated scanning aperture system that selectively admitted light into different locations of a device-coupled multi-ridge ATR crystal.

Independent of the detection system used previous applications of multi-ridge ATR crystals for on-chip measurements have largely relied on patterning photoresist onto the crystal sampling 
surface to define the entire microfluidic device. ${ }^{7,9,10}$ Thus, only the most simplistic microfluidic features could be realized on the available surface area, measuring approximately $1 \mathrm{~cm}^{2}$. Worse, spin-coated photoresists were dominated by edge bead effects, which resulted in non-uniform channel wall height and complicated the attachment of a sealing layer to enclose the channels. As a result, the approach of patterning fluidic features atop the multi-ridge ATR crystals is most amenable to an open channel architecture. In the sole work that was successful in sealing the channels on top of the top-side patterned device, the world-to-chip interface dominated the space requirements, leaving very little space for channels and analyte monitoring. ${ }^{7}$

In the current work we propose a different strategy, whereby the ATR crystal is embedded into the microfluidic device. As we show, isolating the sensing to a specific region on the resulting spectrofluidic device reduces wasted sensing surface area previously used for structural support of the device. This unlocks significant potential for on-chip chemical sensing applications by removing limitations on device sealing, world-to-chip interfacing, fluidic manipulations, and device complexity. We demonstrate the freedom in microfluidic device design by incorporating multi-ridge crystals into 4 fundamentally different device architectures. In addition, we couple the devices with the previously mentioned aperture scanning accessory to conduct proof-of-concept measurements in assaying, continuous scanning during dynamic changes to flow conditions, visualization of concentrations gradients, and to follow on-chip reaction kinetics.

\section{Experimental:}

Materials and solutions 
Solutions of sodium phosphate monobasic (84486-300, Anachemia, Canada) and D-glucose (G8270-1KG, Sigma-Aldrich, Canada) were used as a test analytes. An amount of $2.84 \mathrm{~g}$ of sodium phosphate dibasic $\left(\mathrm{Na}_{2} \mathrm{HPO}_{4}\right)$ and $36.07 \mathrm{~g}$ of $\mathrm{D}$-glucose were dissolved in same volume deionized water (200 mL, DI water), and the resulting concentration were $0.10 \mathrm{M}$ and $1.00 \mathrm{M}$, respectively. Different solutions were obtained by serial dilution using DI water.

\section{Spectrofluidic device materials}

The microchannel layer used in this work were fabricated in polydimethylsiloxane (PDMS) mixed with a cross-linking agent at a ratio of $10: 1$ and then casting against a mould. All moulds were fabricated by photo lithography using laminate photoresist with $50 \mu \mathrm{m}$ height (SF4000, Mungolux, Germany) adhered to glass. Channel geometries were designed by software (AutoCAD, Autodesk, USA). The surface metrology of the embedded ATR sensing layer was measured with an optical profilometer (ContourX-200, Bruker, USA). Multi-ridge ATR crystals (Basic Universal, IRUBIS $\mathrm{GmbH}$, Germany) were used without further modifications.

\section{Fourier Transform Infrared Spectroscopy}

Measurements were collected on either on compact FTIR spectrometer (Alpha II, Bruker Ltd. Canada) controlled by computer software (OPUS, Bruker, USA) or a research grade spectrometer (Nicolet iS50, Thermo Fisher Scientific, USA) with a KBr beam splitter and a nitrogen-cooled MCT detector, and controlled by software (OMNIC, Thermo Fisher Scientific, USA). In both cases a purge gas generator was used to limit atmospheric interference. All spectra were acquired with 32 co-additions and a $4 \mathrm{~cm}^{-1}$ spectral resolution, except where otherwise stated. Spectrofluidic 
devices were interfaced with an ATR spectroscopy microfluidic assay accessory (ASMAA) for spatial-selective measurements which is described elsewhere. ${ }^{9}$ Data analysis was carried out using open source software (Quasar, Orange, ). ${ }^{11}$

Computational fluid dynamics simulations

The flow of liquids in devices were simulated by fluid simulation analysis software (COMSOL Multiphysics 5.4, COMSOL, Sweden)

\section{Fabrication of an ATR-embedded spectrofluidic device:}

The following fabrication protocol outlines a general method for incorporating a planar multiridge ATR crystal with the light coupling into any microfluidic device design (Figure 1), includes the fabrication of two main components, the ATR sensing layer (Figure $1 \mathrm{a}$ ), and the microchannel feature layer (Figure 1b). This is a different approach than used previous to integrate planar detection elements at the base of the channel. ${ }^{12}$ The fabrication of the ATR sensing layer starts by placing the crystal face-down (groove-side up) on a thin polyethylene (PE) film (Plastic wrap, Selection, Canada), which is supported by a glass substrate and set at the bottom of a petri dish (Figure $1 \mathrm{a}(\mathrm{i})$ ). Then, a Teflon plug with a tapered edge of $20^{\circ}$ was pressed against the ridge side. This angle matched the light path angle from the ATR spectroscopy mapping aperture accessory (ASMAA). ${ }^{9}$ To improve the reproducibility and accuracy of the optical access port, a jig was machined that aligns the ATR crystal and the Teflon plug and applies even pressure such that their contact was uniform, leaving no spaces for the pre-cured PDMS to penetrate between them (Figure SX). Then a mixture of liquid PDMS and cross linker (Sylgard184, Dow corning, Canada) at 
a 10:1 ratio was poured on top of the crystal (Figure 1a(ii)) until the level was approximately 1 $\mathrm{mm}$ higher than the backside and cured at $70{ }^{\circ} \mathrm{C}$ for $4 \mathrm{~h}$. After the PDMS solidified, the Teflon plug was removed, aided by its tapered edge. In some cases, a small meniscus extended along the tapered edge of the plug and remained after solidification (Figure 1(iii)). The result is a sensing layer with an optical access port with sloped walls to accommodate incoming light from the spectrometer source without causing ATR ridge side. The opposite side features a completely planar PDMS/ATR surface for bonding to the microfluidic feature side with the ATR being aligned with the characterisation component. The microfluidic feature layer was created with the use of a photoresist mould, which was created using a lamination method as described elsewhere(Figure $1 \mathrm{~b}(\mathrm{i}))^{13}$. The same PDMS and cross-linker mixture was poured on top of mould cured at $70{ }^{\circ} \mathrm{C}$ (Figure $1 \mathrm{~b}$ (ii)). After removal of the microchannel feature layer from the mould, inlet and outlet access holes were punched (Figure 1b (iii)). Finally, the microchannel layer and the ATR sensing layer were aligned and bonded after exposure to plasma gas (PDC-001, Harrick Plasma, USA) (Figure $1 \mathrm{c}$ ). Alignment was achieved by either cross hair marks or the edges of channel in the microchannel feature layer that matched the ATR crystal corners.

The spetrofluidic devices in this work were imposed atop of a mirror assembly, which redirected light from the transmission sample compartment into the device from the bottom-side where the ATR ridges were exposed. In all cases the liquid direction was parallel to the optical axis of the instrument. 
(a)

(i)
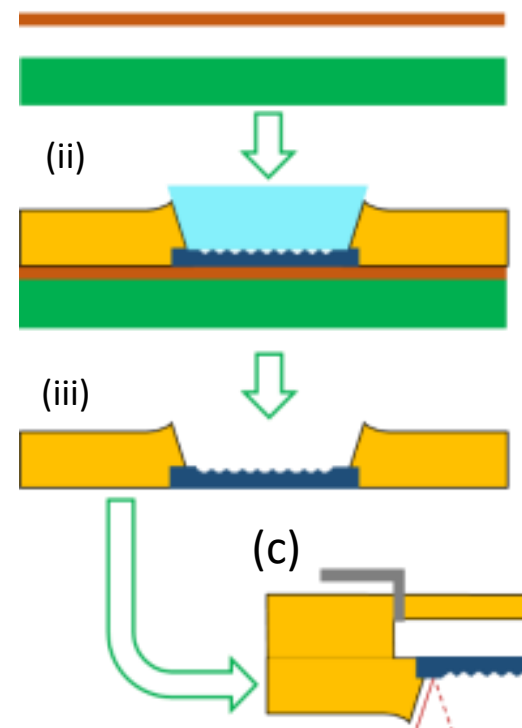

(c) (b)

(i)

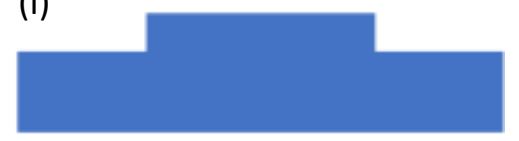

(ii)

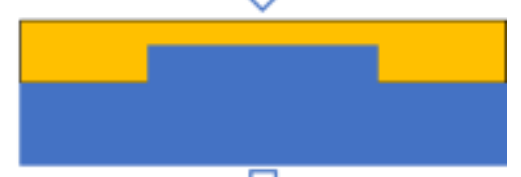

(iii)

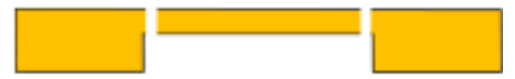

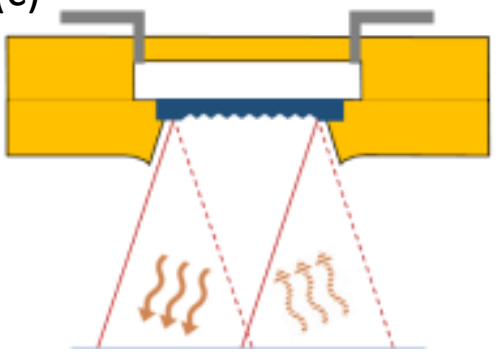

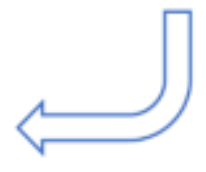

Figure 1. A Schematic image of the fabrication process for the ATR sensing layer (a), microfluidic channel layer (b), and the final spectrofluidic device (c). In total, 4 different spectrofluidic devices were created, each with a purpose- specific microfluidic channel layer.

\section{Results and discussion:}

\section{A device for high sensitivity measurements}

The first device design takes advantage of an enlarged footprint to accommodate two inlets and one outlet, which were located away from the ATR sensing region (Figure 2a). The ATR crystal was fully enclosed within a single microchannel of width of $12 \mathrm{~mm}$ which enabled the entire crystal surface to directly contact the channel contents, thereby providing the highest interface area 
between the probing evanescent fields at analyte, and the highest potential sensitivity. Analyte liquids were introduced into the device by syringe pump with separate solutions connected to each inlet (Figure 2b). We alternatively admitted $\mathrm{Na}_{2} \mathrm{HPO}_{4}$ solution $(0.1 \mathrm{M})$ and water into the channel through one of the two inlets, and sequentially obtained the respective spectra (Figure 2c). The bands at 1072 and $990 \mathrm{~cm}^{-1}$ belong to the stretching vibration of $v 1\left(\mathrm{PO}_{4}\right)$ and $v 1\left(\mathrm{PO}_{3}\right)$, respectively. Next, we acquired data while flow manipulations were underway. In order to scan as quickly as possible, we took advantage of the device high sensitivity so that we could minimize the number of scans to observe rapidly changing signals. In the next experiment, we alternatively admitted water and $\mathrm{Na}_{2} \mathrm{HPO}_{4}$ solution into the channel at a high flow rate $\left(10 \mathrm{~mL} \cdot \mathrm{h}^{-1}\right)$ for 90 for each liquid. Simultaneously, spectra were collected at a high acquisition rate. The instrument base time to obtain a spectrum was $5 \mathrm{~s}$ due to overheads in communication, calculations, and data management. As we could scan twice in this time, we set the number of scans to 2 , and obtained time-series spectra with a time-resolution of $5 \mathrm{~s}$. Integration of the peak for $\mathrm{Na}_{2} \mathrm{HPO}_{4}$ provided the composition of flow in channel versus time (Figure 2d). Based on Eqn 1, the velocity of liquids in channel was $5 \mathrm{~mm} \cdot \mathrm{s}^{-1}$. In the $5 \mathrm{~s}$ between measurements, the fluid can move $25 \mathrm{~mm}$, which is greater than the $9 \mathrm{~mm}$ length of the downstream dimension of the ATR crystal. Therefore, it expected that once the new liquid reaches the ATR crystal, its spectral signature will jump from zero to maximum intensity between 2 consecutive data points. The result indicated the process of flow switching in device characterization zone was less than $5 \mathrm{~s}$ at this flow rate.

$$
\begin{aligned}
& v\left(\mathrm{~mm} \cdot \mathrm{s}^{-1}\right)=\mathrm{Q}\left(\mathrm{mL} \cdot \mathrm{h}^{-1}\right) / \mathrm{A}\left(\mathrm{cm}^{2}\right) \\
& t\left(\mathrm{~s}^{-1}\right)=\mathrm{d}(\mathrm{cm}) / v\left(\mathrm{~cm} \cdot \mathrm{s}^{-1}\right)
\end{aligned}
$$


Next, we generated a calibration curve and determined the limit of detection (LOD). Analyte solutions were $\mathrm{Na}_{2} \mathrm{HPO}_{4}$ and glucose solution. Phosphate is a major component in the popular buffer, phosphate-buffered saline (PBS), which has wide used in biological studies. ${ }^{14}$ Glucose exists in different biofluids in our bodies at a wide range of concentrations. ${ }^{15}$ Lowering the LOD of glucose could expand the clinical applications to other body fluids where glucose concentrations are lower than in the in blood glucose range, such as urine, saliva, tears, sweat, and interstitial fluids. ${ }^{16}$
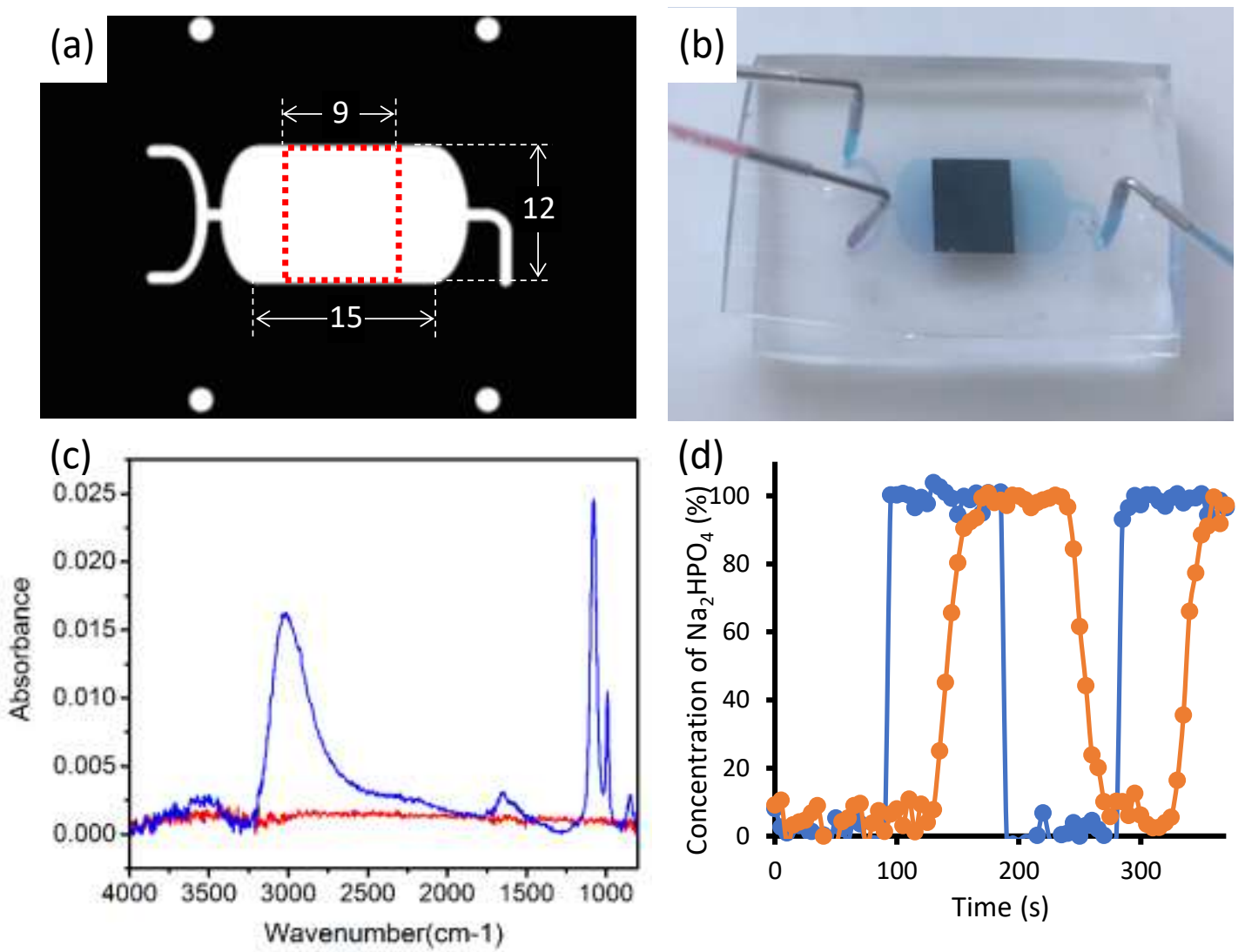

Figure 2. Schematic (a) and image (b) of the 2 inlet and 1 outlet design. Dimensions are in millimeters. Image in (b) uses blue and red food dyes to mark different liquid streams, with blue being admitted during the photo. (c) Individual spectra of water (red) and disodium phosphate (blue) (water as background). (d) Result from alternating flow of water and disodium phosphate. 
The spectra to calculate the integration value were acquired with 2 co-additions and an $8 \mathrm{~cm}^{-1}$ spectral resolution.

To demonstrate quantitative analysis ability of this device, two chemicals, disodium phosphate $\left(\mathrm{Na}_{2} \mathrm{HPO}_{4}\right)$ and D-glucose, were selected for calibration curve characterization. The calibration curve is the linear relationship between spectral peak intensity and concentration of the analyte. These two chemicals both have broad application in studying biological assays. $\mathrm{Na}_{2} \mathrm{HPO}_{4}$ is the major constituent of phosphate-buffered saline (PBS), a common buffer solution for studying microbial sample in microfluidics ${ }^{17} \mathrm{D}$-glucose is also a common composition in many nutrient solutions as the energy source for cultivating microbes. ${ }^{18}$

The deionized water which was the solvent for sample solution was used as the background sample here. Both the water and solution were loaded onto the open channels by dropping from a pipette. After the background was acquired, the deionized water was removed from the surface first by a swapping cotton. Then, the open channel was rinsed and cleaned with following analyte 3 times before next measurement.

The spectra processing and peak integral calculation were carried out on a data visualizing software, Orange, developed by Bioinformatics Laboratory from University of Ljubljana, Slovenia. A linear baseline correction was first deployed using $1400 \mathrm{~cm}^{-1}$ and $800 \mathrm{~cm}^{-1}$ as reference points. This was followed by a gaussian smoothing with standard deviation as 4 . For glucose, the peak centered at $1100 \mathrm{~cm}^{-1}$ was integrated from 930 to $1180 \mathrm{~cm}^{-1}$, which is assigned as the C-O-C stretching of glucose backbone ${ }^{19}$ For $\mathrm{Na}_{2} \mathrm{HPO}_{4}$, the peak centered at $990 \mathrm{~cm}^{-1}$ was integrated from 964 to $1003 \mathrm{~cm}^{-1}$, which was assigned as P-O asymmetric stretching ${ }^{20}$ The integral data were then transfer to Microsoft Excel for linear regression and plotting. 
The calibration curve suggests, for this experiment setup, the limit of detection (LOD) for glucose is approximately at $3 \mathrm{mM}$. This is an improved result comparing to the previous study using a similar setup, in which the LOD was approximately at $27 \mathrm{mM} .{ }^{9}$ We attribute the improvement to the ridge orientation that was perpendicular to the optical axis and the enlarged surface area that was in contact with the liquid. We note that the band area stopped decreasing at this concentration indicating that the limit of detection was being driving by an adsorbed D-glucose layer at the surface, rather than a limit of detection in the bulk liquid. The limit of detection for $\mathrm{Na}_{2} \mathrm{HPO}_{4}$ was only $1 \mathrm{mM}$ and did not appear to be limited by an adsorbate, as the HPO3 ions is smaller and does not participate in non-specific adsorption such as large organic molecules like D-glucose.(ref)

Interestingly, the calibration curve of glucose, unlike that of $\mathrm{Na}_{2} \mathrm{HPO}_{4}$, does not converge with the $y$ axis at 0 value. One possible explanation is the adsorption of analyte to the ATR crystal. Since the crystal used here is silicon, a thin layer of oxide will form spontaneously ${ }^{21}$ on the side contacting with the analyte, which will therefore interact with the analyte. This was the case for water as shown in our previous study, where the broad peak of $\mathrm{O}-\mathrm{H}$ stretching centered at lower frequency compared to standard spectrum from NIST library5. Consequently, because D-glucose molecule is rich in hydroxyl group, the molecule can interact wit the surface via hydrogen bond. This enriches the molecule concentration at vicinity of crystal-solution interface from the rest of the bulk, leading to the constant response of detection of glucose at low concentration. 

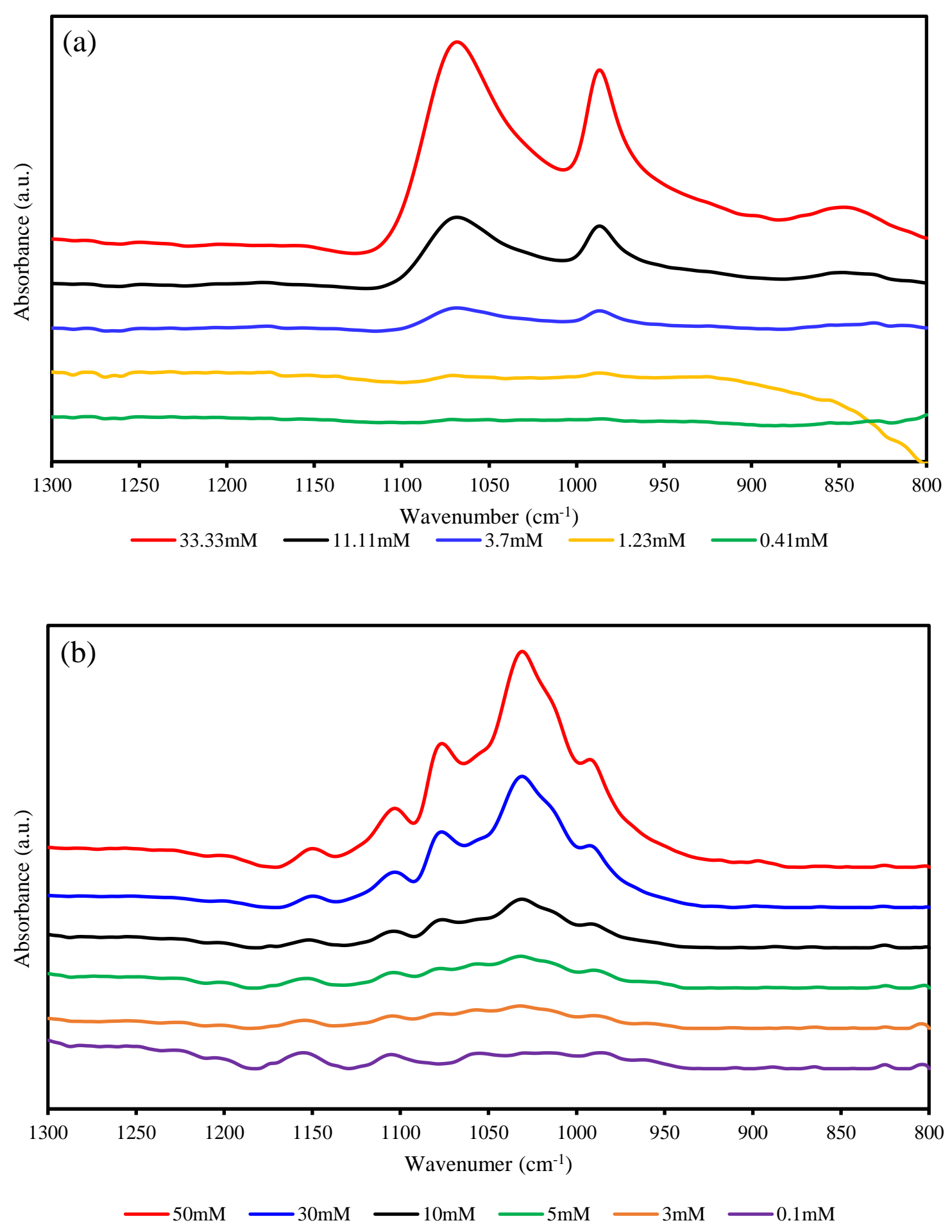


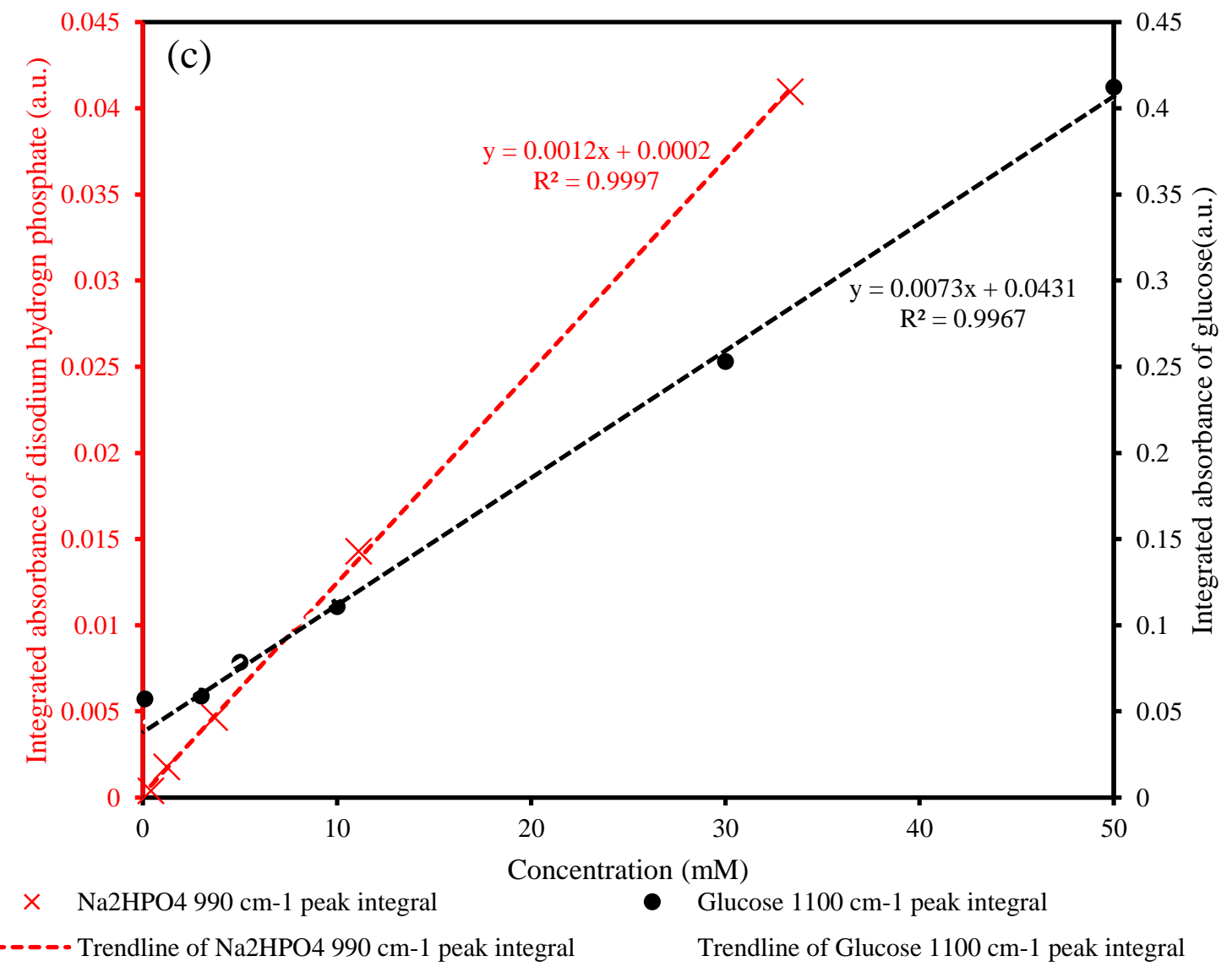

Figure 3. FTIR spectra of (a) $\mathrm{Na}_{2} \mathrm{HPO}_{4}$ and (b) D-glucose at different concentration and (c) calibration curve of abovementioned two chemicals based on the integral of corresponding characteristic peaks.

\section{Spectrofluidic devices designed for spatially resolved measurements}

In the remainder of the paper, the presented spectrofluidic devices analysed on a new mapping accessory called an ATR spectroscopy mapping aperture accessory (ASMMA) ${ }^{9}$. In sub-selecting the region of interest using an aperture, a key consideration is the orientation of the ridges relative to the aperture. There are two choices for embedding ATR crystals into the spectrofluidic devices. In the first, the ridges are along the $x$-direction (perpendicular to the aperture) and in the second, 
the ridges are along the $y$-direction in the second (parallel to the aperture). The two orientations are shown in Figure 4a. We filled each channel with water and scanned the ASMAA aperture along the $x$-direction. As observed in Figure 4b, the difference in ATR ridge orientation has a significant impact on both the light intensity and the water absorbance profiles when coupled with the scanning aperture. In the first case when the ridges are positioned along the $\mathrm{x}$-direction, an $\mathrm{x}$-scan of the moving aperture produced a continuous change in intensity, which peaked near the centre of the device and tailed off slightly at the edges with an approximately Gaussian profile (Figure 4c). In the case when the ATR ridges were oriented along the y-direction, a series of oscillations in the intensity and absorbance values were observed as a function of the aperture displacement, which were enveloped with a similar Gaussian profile (Figure 4c). Moreover, we drop a water on top of crystal, and repeat $\mathrm{x}$-scan twice with ATR ridges oriented along the $\mathrm{x}$ - and $\mathrm{y}$ - direction, successively. By integrating the peak at $3300 \mathrm{~cm}^{-1}$ of each spectrum, we obtained 2 cross-section plot profile curves which exhibited the same shape (Figure 4d). The latter behaviour was observed previously using an aperture scanning approach in the same orientation ${ }^{9}$ and as well using a scanning synchrotron beam ${ }^{7}$ and is related to light lost to refracted light being split into divergent paths, which are then blocked by the aperture plate on their way out. In this case, aligning channels along the ridges, can take advantage of peaks in light throughput, could be advantageous. However, in the case where the channels oriented in the x-direction, ...

The ASMAA controls the position of an aperture, which admits light to specific, computercontrolled positions on the ATR crystal, within the spectrofluidic device. Guided by the goal to have the most flexible fabrication protocol possible, the ATR crystal can be imbedded in the channel with ridges parallel or perpendicular to the direction of flow and/or the optical beam path. 

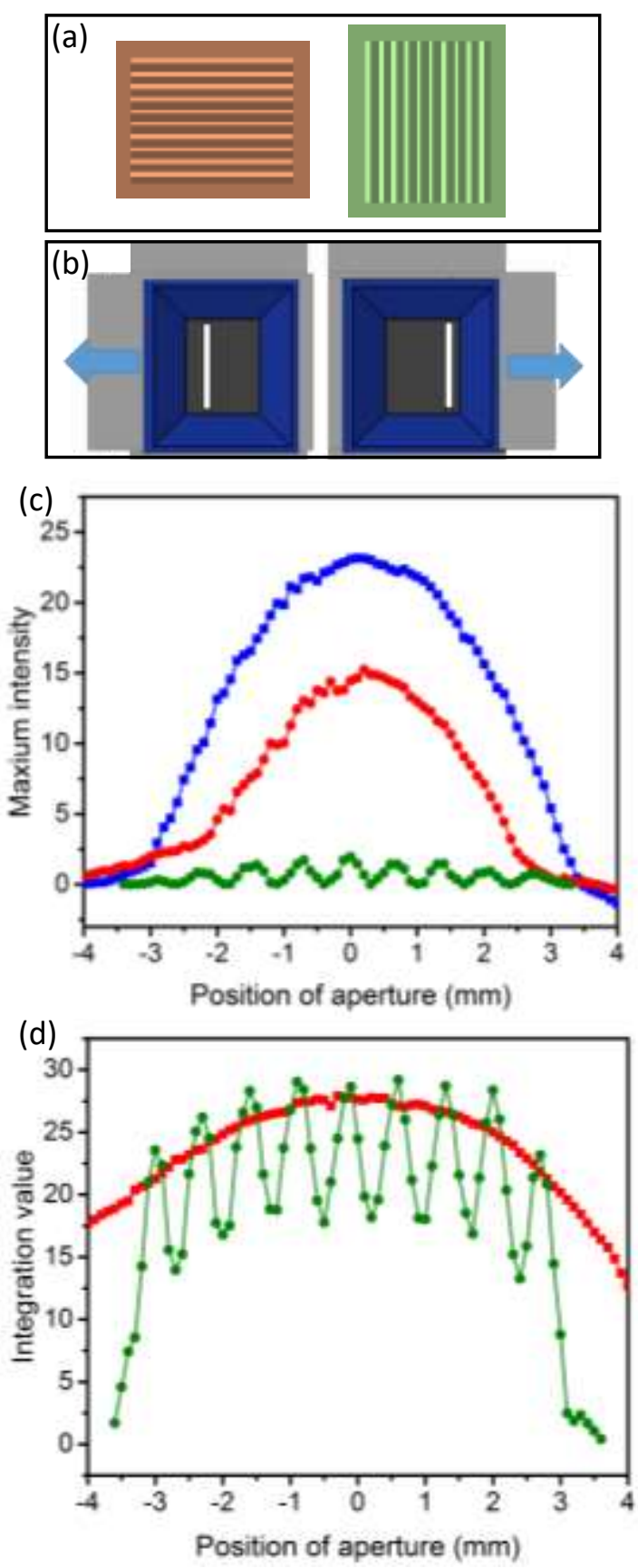

Figure 4 Two ATR ridge orientations used in this study are (a) perpendicular and (b) parallel to the aperture long edge (oriented along the $y$-direction). (c) Intensity versus $x$-position during an x-scan with crystal surface down (blue) and ridges being in the perpendicular to the aperture long edge (orange squares) (red) and parallel to the aperture long edge (green circles) (green). (d) Integration value of absorbance peak at $3300 \mathrm{~cm}^{-1}$ of water versus $\mathrm{x}$-position during an $\mathrm{x}$ scan with at same condition as (c). The width of aperture is $0.4 \mathrm{~mm}$. 


\section{Linear scanning parallel to ridges using a 6-inlet spectrofluidic device}

We demonstrate the importance a larger device footprint to accommodate on arbitrary number of world-to-chip connections for studies of complex on-chip laminar co-flow patterns. Such patterns have been used extensively in our group and elsewhere to present liquid-liquid interfaces for spatially selective precipitation processes, ${ }^{22}$ reactions, ${ }^{23}$ creation of concentration gradients, ${ }^{24}$ and to create nutrient rich/poor regions for patterned growth of microbial samples. ${ }^{25}$

The design (Figure 5a), featuring six inlets and one outlet, is designed to generate laminar co-flow patterns in main channel. Inlet 1 is divided into 2 channels, providing a sheath flow along the main channel walls, thus confining the other 5 streams to the middle position of the channel (Figure 5b). The ridge direction was perpendicular to the aperture. We chose this orientation so we could have continuous 1-D scan maps without oscillations signals that are obtained for the other orientation (as seen in Figure 4). In our test, we pumped distilled water into Inlet $1\left(12 \mathrm{~mL} \cdot \mathrm{h}^{-1}\right)$ and into Inlets 3 and $5\left(3 \mathrm{~mL} \cdot h^{-1}\right)$. An aqueous solution of $\mathrm{Na}_{2} \mathrm{HPO}_{4}(0.1 \mathrm{M})$ was pumped into in Inlets 2, 4 and 6 at same flow rate $\left(3 \mathrm{~mL} \cdot \mathrm{h}^{-1}\right)$. A separate syringe was attached to each inlet to ensure there was no flow redirection. The elevated total flow rate of $Q_{T}=Q_{1}+Q_{2}+Q_{3}+Q_{4}+Q_{5}+Q_{6}=27$ $\mathrm{mL} \cdot \mathrm{h}^{-1}$ was operated for 4 hours without leaking. The same device maintained its integrity during repeated operation under similar flow rates for over a month, demonstrating the robust nature of the embedded ATR crystal. A 6-flow interface was established as seen in Figure 5b. A COMSOL simulation was using the same experimental flow conditions (Figure 5c) predicted the same flow pattern as observed in real device. Then, we applied a $0.4 \mathrm{~mm}$ aperture scan the device from one side of the device to the other with step of $0.1 \mathrm{~mm}$. Integration of $\mathrm{Na}_{2} \mathrm{HPO}_{4}$ signature bands at $1072 \mathrm{~cm}^{-1}$ provided the chemical composition of flow streams across channel (Figure $5 \mathrm{~d}$ ). The curve shows three flow streams comprised of $\mathrm{Na}_{2} \mathrm{HPO}_{4}$ from inlets 2, 4 and 6, each separated by 
a flow of distilled water. The integration area of $\mathrm{Na}_{2} \mathrm{HPO}_{4}$ absorbance bands was maximum in the centre and became reduced value in the wings despite each stream having the same concentration (Figure $5 d$ ). This was expected from previous studies due to a combination of the Gaussian IR beam profile provided by the spectrometer and the presence of some constant background signal reaching the detector. ${ }^{9}$

(a)

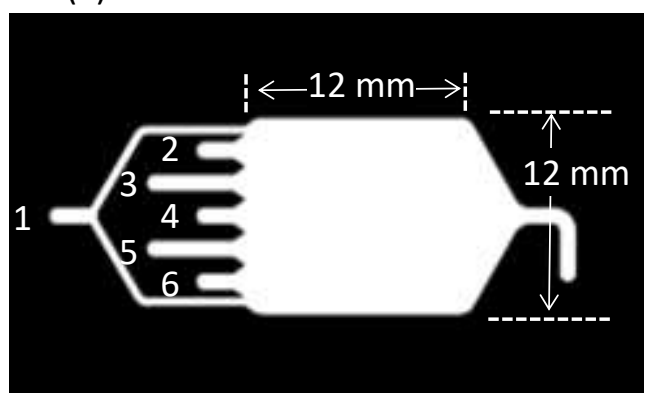

(c)

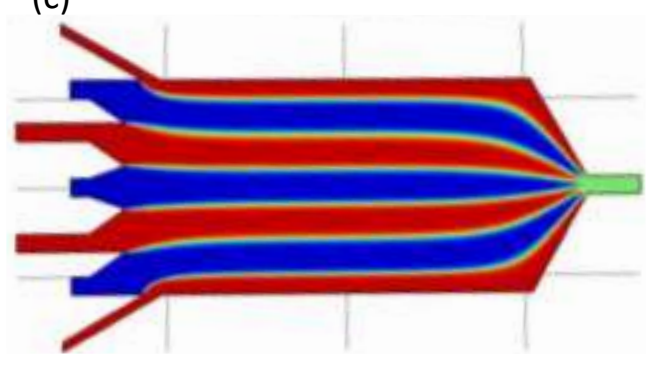

(b)

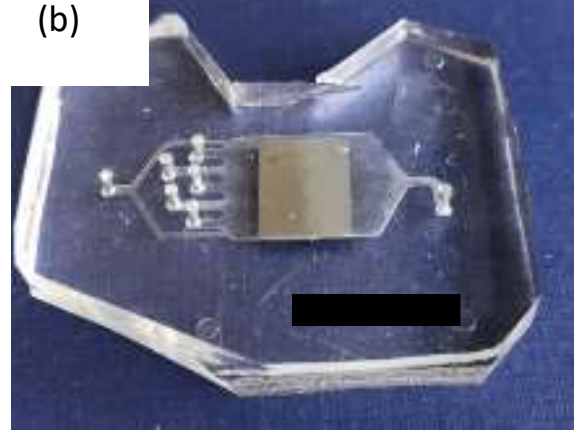

(d)

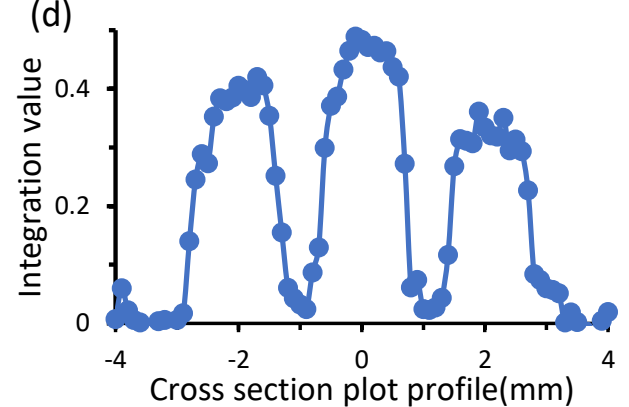

Figure 5. a) Schematic of the multichannel co-flow device assay device with alignment marks. (b) Image of the multichannel co-flow device. (c) Results from flow simulation. (d) Profile of absorbance peak intensity at $1072 \mathrm{~cm}^{-1}$ of disodium phosphate. The ATR ridge direction was perpendicular to the aperture.

\section{A 9 channel spectrofluidic assay device}

In the next example, we demonstrate a spectrofluidic device, which aligns the channels with the direction of the ATR ridges, for conducting assay experiments. The microchannel feature layer contained 9 parallel channels (Figure 6a) each with a channel width of $0.4 \mathrm{~mm}$ and a $0.3 \mathrm{~mm}$ wall separating adjacent channels. Therefore, the centre-lines of each channel and ridge had the 
same periodicity of $0.7 \mathrm{~mm}$, and the proper alignment resulted in the centre-line of each channel being matched to that of each ridge in the final spectrofluidic device (Figure 6b). The ASMAA was used to control the position of a $0.4 \mathrm{~mm}$ aperture such that it could jump to predefined positions corresponding to the mid-point of each channel/ridge assembly. As determined previously via simulation, an aperture width of $0.4 \mathrm{~mm}$, translates to measurement zone width of $0.2 \mathrm{~mm}$ at the sample surface. ${ }^{9}$ It is understood that advantage of parallel ridge to aperture is the ability to admit more light into small channels. Thus, the measurement zone is expected to be centred within the channel, with no interference from either PDMS channel wall. A background spectrum was acquired from each channel/ridge mid-position, when the channels were filled with dye water. As a proof-of-concept, the evaluation of each separate channel content was verified using $0.1 \mathrm{M} \mathrm{Na}_{2} \mathrm{HPO}_{4}$ solution admitted to channels $1,3,5,7$, and 9 while pure water was admitted to channels 2, 4, 6, and 8. Each inlet was connected via a separate syringe pumping at a flow rate of $\mathrm{Q}=0.5 \mathrm{~mL} \cdot \mathrm{h}^{-1}$. Spectra were obtained from each channel by stepping the aperture by $0.7 \mathrm{~mm}$ increments. Result showed the expected spectral signatures from the $\mathrm{Na}_{2} \mathrm{HPO}_{4}$ solution while the channels that contained water showed no bands due to the background scan matching that of the sample (Figure 6c). Moreover, the water-containing flow streams showed no spectral contamination from the $\mathrm{Na}_{2} \mathrm{HPO}_{4}$ solution. The absorbance area for the $\mathrm{Na}_{2} \mathrm{HPO}_{4}$ characteristic band $\left(1072 \mathrm{~cm}^{-1}\right)$ was analysed for each channel (Figure $6 \mathrm{~d}$ ). The lack of any spectral contamination from the $\mathrm{Na}_{2} \mathrm{HPO}_{4}$ solution in the water channels indicates that there were no leaks between channels nor any cross between their measurements. A similar experiment was run, but this time using a dry background and water 
and DMF solutions showing 9 spectra, verifying the generality of the technique and providing a more robust validation of leak-proof operation (ESI Figure S4)

(a)

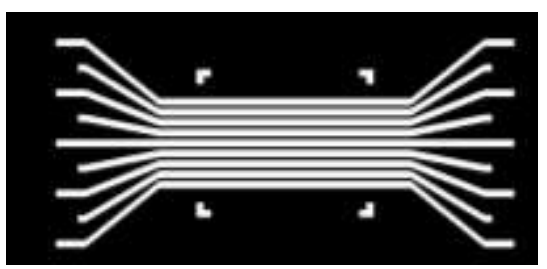

(c)

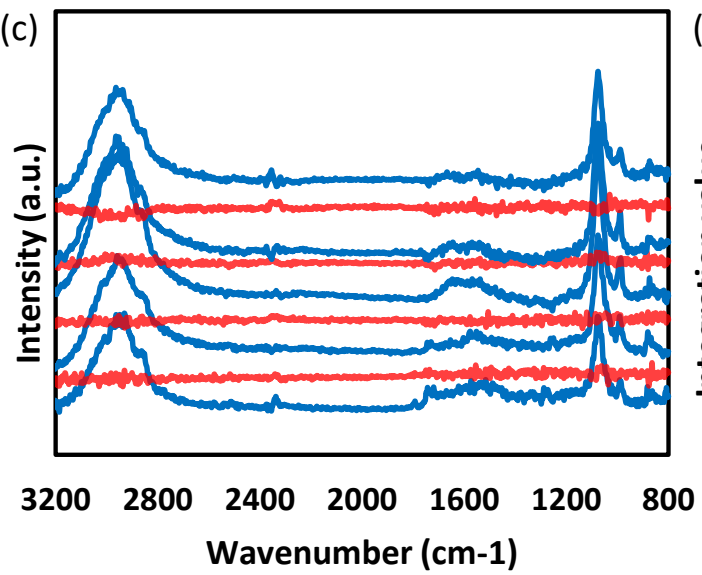

(b)
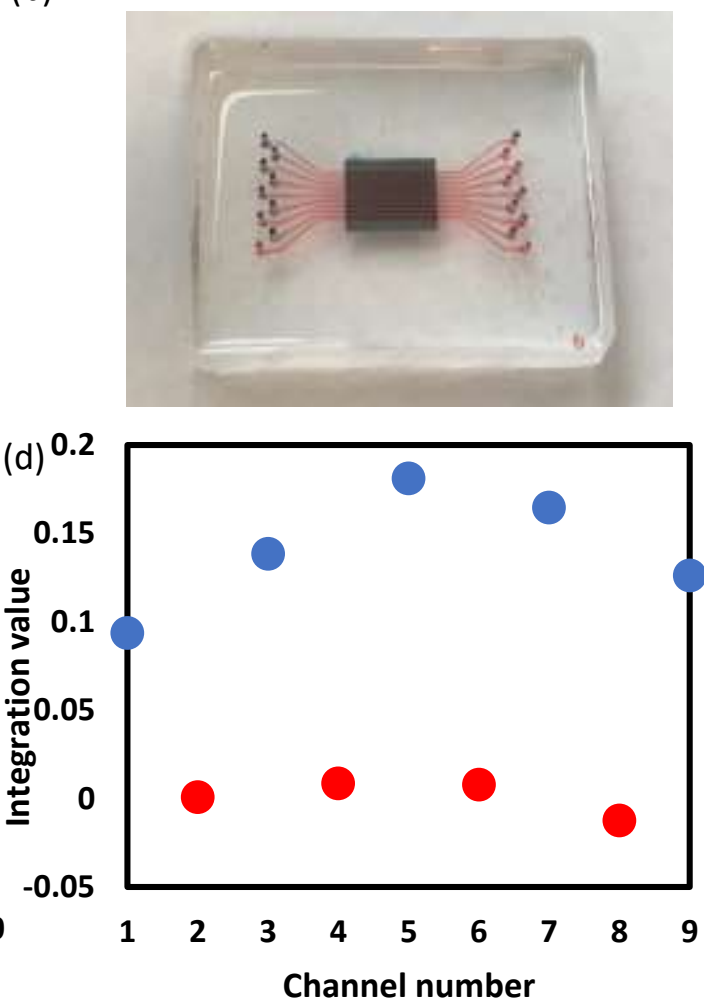

Figure 6. (a) Schematic of a 9 channel assay device with alignment marks to ensure each channel overlap with ridges. (b) Image of 9 channels assay device. (c) Spectra obtained in each channel using water as background. (d) Profile of absorbance peak intensity at $1072 \mathrm{~cm}^{-1}$.

\section{A spectrofluidic reactor for chemical kinetics}

Next we demonstrated a 2-inlet spectofluidic device design to study reaction kinetics. After entering the device, fluid streams were led to a zigzag mixing channel with reduced width (100 $\mu \mathrm{m})$ before entering a reaction monitoring compartment atop the multi-ridge ATR crystal. The reaction monitoring chamber consisted of a series of switchback which were co-linear to the 
ATR ridges on the opposite side of the wafer. Channel dimensions were enlarged to $0.7 \times 0.05$ $\mathrm{mm}$ to provide more contact with the ATR surface. The switchback design (Figure 7a) totaled 10 parallel flow paths, the first 9 of which were coupled to each of the ATR ridges (Figure 7b). Thus, all ridges were coupled to channels, in order to maximize the usable sensing surface on the ATR crystal. Changing the position of aperture, admitted light to a different ridge/channel location on-chip to track the reaction state at different position in the channel. With the knowledge of the fluid velocity that was calculated based on the volumetric flow rate and the channel crosssectional area (A) (Eqn. 1) and the mid-point position of each channel switchback, a distance to time transformation (Eqn. 2) was used to establish the time-base for the monitored reaction kinetics.

Before the experiment, we used the same 9 channel assay device shown in Figure 5 to record spectra of $\mathrm{Na}_{2} \mathrm{HPO}_{4}$ in $\mathrm{pH}$ solutions ranging from 1.5 to 9 (Figure 7c). The concentration of $\mathrm{HPO}_{4}{ }^{2-}$ at different $\mathrm{pH}$ could be calculated by using the Eqns. 3-5. By integrating the $990 \mathrm{~cm}^{-1}$ absorbance band area we obtained calibration curves which exhibit the relations between integration value and concentration of $\mathrm{HPO}_{4}{ }^{2-}$ and different $\mathrm{pH}$, respectively (Figure $7 \mathrm{~d}$ ).

$$
\begin{array}{ll}
\mathrm{H}_{3} \mathrm{PO}_{4}(\mathrm{~s})+\mathrm{H}_{2} \mathrm{O}(\mathrm{I}) \rightleftharpoons \mathrm{H}_{3} \mathrm{O}^{+}(\mathrm{aq})+\mathrm{H}_{2} \mathrm{PO}_{4}^{-}(\mathrm{aq}) & K_{a 1}=7.11 \times 10^{-3} \\
\mathrm{H}_{2} \mathrm{PO}_{4}^{-}(\mathrm{aq})+\mathrm{H}_{2} \mathrm{O}(\mathrm{I}) \rightleftharpoons \mathrm{H}_{3} \mathrm{O}^{+}(\mathrm{aq})+\mathrm{HPO}_{4}{ }^{2-}(\mathrm{aq}) & K_{a 2}=6.32 \times 10^{-8} \\
\mathrm{HPO}_{4}{ }^{2-}(\mathrm{aq})+\mathrm{H}_{2} \mathrm{O}(\mathrm{I}) \rightleftharpoons \mathrm{H}_{3} \mathrm{O}^{+}(\mathrm{aq})+\mathrm{PO}_{4}{ }^{3-}(\mathrm{aq}) & K_{a 3}=4.49 \times 10^{-13}
\end{array}
$$

Next, we flowed $\mathrm{HCl}$ solution $(0.1 \mathrm{~mol} / \mathrm{L}, \mathrm{pH}=1)$ to Inlet 1 and $\mathrm{Na}_{2} \mathrm{HPO}_{4}$ solution $(0.1 \mathrm{M})$ to Inlet 2. Then we scan 7 parts of the device and integrated the band at $990 \mathrm{~cm}^{-1}$ (Figure $6 \mathrm{e}, \mathrm{f}$ ). The decreasing of the integrated peak intensity suggests that $\mathrm{pH}$ of mixture solution is increasing down the length of the channel, indicating that the phosphate buffer molecular continually capture protons in channel. 


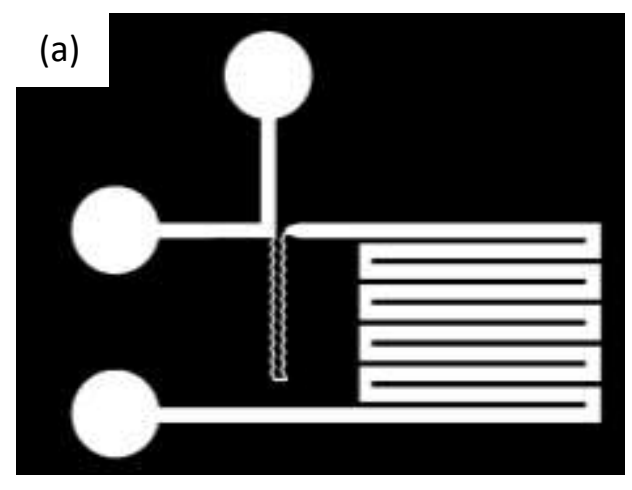

(b)
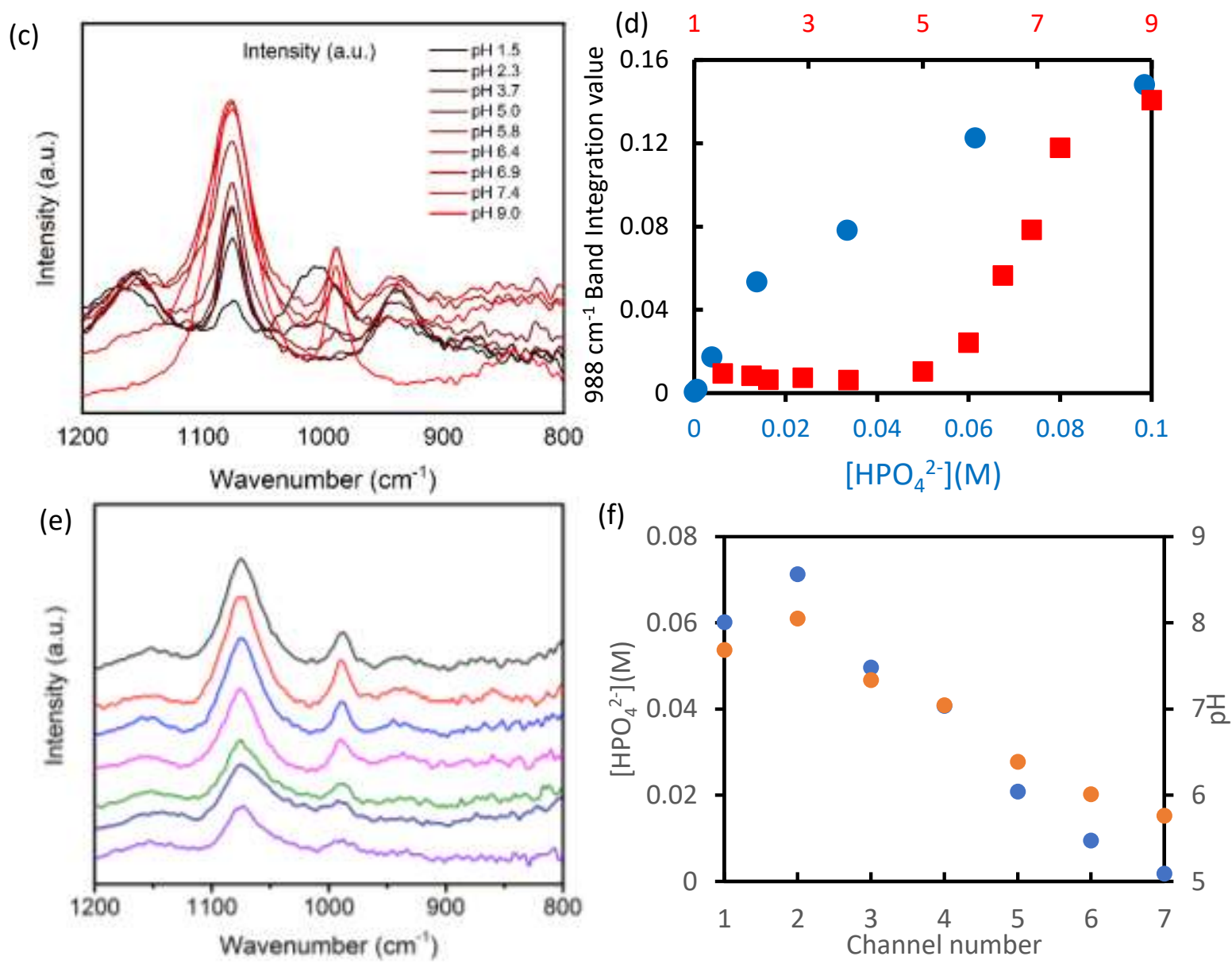

Figure 7. (a) Schematic image of reactor device. (b) A real device with coloured liquid being passed through each inlet channel, with the fully mixed liquid leaving the device. (c) Stacked spectra with changing $\mathrm{pH}$. (d) Integration values of peak intensity at $990 \mathrm{~cm}^{-1}$ of phosphate buffer in different $\mathrm{pH}$ environment with different concentration of $\mathrm{HPO}_{4}{ }^{2-}$. (e) Spectra obtained 
from each channel during reaction. Spectra from top to bottom corresponded to channel 1 to channel 7. (f) Calculated concentration of $\mathrm{HPO}_{4}{ }^{2-}$ and $\mathrm{pH}$ in channel 1 to channel 7 using the integration values of peak intensity at $988 \mathrm{~cm}^{-1}$. The width of aperture is $0.4 \mathrm{~mm}$.

\section{Conclusion}

A novel fabrication method was devised to integrate a silicon multi-ridge ATR element into a microfluidic device, resulting in a generalizable tool for lab-on-chip. The approach was tested on four spectrofluidic device deisgns to obtain deeper working knowledge on multiridge ATR crystals for microfluidics, and to demonstrate sensing applications in complex flow conditions (dynamic flow, multi co-flow interfaces), assaying, and reaction kinetics.

\section{References}

1 (a) K. L. A. Chan, X. Niu, A. J. de Mellob and S. G. Kazarian. Lab Chip, 2010, 10, 2170-2174; (b) B. Lehmkuhl, S. D. Noblitt, A. T. Krummela and C. S. Henry. Lab Chip, 2015, 15, 4364-4368; (c) HY. N. Holman, R. Miles, Z. Hao, E. Wozei, L. M. Anderson, and H. Yang. Anal. Chem., 2009, 81, 8564-8570; (d) L. M. Meireles, I. D. Barcelos, G. A. Ferrari, P. A. A. de A. Neves, R. O. Freitas and R. G. Lacerda. Lab Chip, 2019, 19, 3678-3684.

2 J. Greener, B. Abbasi and E. Kumacheva. Lab Chip, 2010, 10, 1561-1566.

3 D. Voicu, C. Scholl, W. Li, D. Jagadeesan, I. Nasimova, J. Greener and E. Kumacheva. Macromolecules, 2012, 45, 4469-4475.

4 J. Greener, E. Tumarkin, M. Debono, C-H. Kwan, M. Abolhasani, A. Günther and E. Kumacheva. Analyst, 2012, 137, 444-450.

5 M. Pousti and J. Greener. Surf. Sci., 2018, 676, 56-60. 
6 (a) K. L. A. Chan, X. Niu, A. J. de Mello and S. G. Kazarian. Anal. Chem., 2011, 83, 3606-3609; (b) K. L. A. Chan, S. Gulati, J. B. Edel, A. J. de Mello and S. G. Kazarian. Lab Chip, 2009, 9, 2909-2913.

7 T. A. Morhart, S. T. Read, G. Wells, M. Jacobs, S. M. Rosendahl, S. Achenbach and I. J. Burgess. Anal. Methods, 2019, 11, 5776-5783.

8 C. Kratz, A. Furchner, G. Sun, J. Rappich and K. Hinrichs. J. Phys.: Condens. Matter. 2020, 32, 393002

9 M. Joly, T. Deng, T. A. Morhart, G. Wells, S. Achenbach, A. Bégin-Drolet and J. Greener. Anal. Chem., 2021, 93, 14076-14087

10 T. A. Morhart, S. Read, G. Wells, M. Jacobs, S. M. Rosendahl, S. Achenbach and I. J. Burgess. Appl. Spectrosc., 2018, 72, 1781-1789.

11 (a) M. Toplak, S. T. Read, C. Sandt and F. Borondics. Cells, 2021, 10, 2300. (b) J. Demsar, T. Curk, A. Erjavec, C. Gorup, T. Hocevar, M. Milutinovic, M. Mozina, M. Polajnar, M. Toplak, A. Staric, M. Stajdohar, L. Umek, L. Zagar, J. Zbontar, M. Zitnik, B. Zupan. J. Mach. Learn. Res., 2013 $14,2349-2353$.

12 M. P. Zarabadi, F. Paquet-Mercier, S. J. Charette, and J. Greener. Langmuir, 2017, 33, 20412049.

13 F. Asayesh, M. P. Zarabadi, N. B. Aznaveh and J. Greener. Anal. Methods, 2018, 10, 45794587.

14 J. Kang, S. Ramu, S. Lee, B. Aguilar, S. K. Ganesan, J. Yoo, V. K. Kalra, C. J. Koh, and Y-K. Hong. Anal. Biochem., 2009, 386, 251-255.

15 L. Boselli, T. Pomili, P. Donati and P. P. Pompa. Materials, 2021, 14, 1978.

16 (a) J. Heikenfeld, A. Jajack, B. Feldman, S. W. Granger, S. Gaitonde, G. Begtrup and B. A. Katchman. Nat. Biotechnol., 2019, 37, 407-419; (b) W. Zhang, Y. Du and M. L. Wang. Sens. BioSens. Res., 2015, 4, 23-29. 
17 S. Miller, A. A. Weiss, W. R. Heineman and R. K. Banerjee. Sci. Rep., 2019, 9, 14228.

18 W. Keenleyside. Microbiology: Canadian Edition, 2019.

19 M. Ibrahim, M. Alaam, H. El-Haes, A. F. Jalbout and A. de Leon. Eclet. Quim., 2006, 31, 15-21.

20 Y. Arai and D. L. Sparks. J. Colloid Interface Sci., 2001, 241, 317-326.

21 C. Bohling and W. Sigmund. Silicon, 2016, 8, 339-343.

22 N. Jia, E. Rosella, E. Juère, R. Pouliot, F. Kleitz and J. Greener. Lab Chip, 2020, 20, 1066-1071.

23 E. Kang, S-J. Shin, K. H. Lee and S-H. Lee. Lab Chip, 2010,10, 1856-1861.

24 E. Berthier and D. J. Beebe. Lab Chip, 2014, 14, 3241-3247.

25 (a) N. B. Aznaveh, M. Safdar, G. Wolfaardt and J. Greener. Lab Chip, 2014, 14, 2666-2672; (b)

M. Rothbauer, P. Ertl, B. A. Theiler, M. Schlager, U. B. Sleytr and S. Küpcü. Adv. Mater.

Interfaces, 2015, 2, 1400309 
SUPPORTING INFORMATION

Disposable spectrofluidic devices for attenuated total reflection infrared spectroscopy: characterization sensitivity, spatial resolution and generally applicable to multiple device types.

Nan Jia, ${ }^{\text {a }}$ Arthur Daignault-Bouchard, ${ }^{\mathrm{b}}$ Tianyang Deng, ${ }^{\text {a }}$ Leon Torres de Oliveira, ${ }^{\mathrm{a}}$ André BéginDrolet $^{\mathrm{b}}$ and Jesse Greener ${ }^{\mathrm{a}, \mathrm{c}}$

a Département de chimie, Faculté des sciences et de génie, Université Laval, Québec, QC G1V 0A6, Canada

${ }^{\text {b }}$ Département de génie mécanique, Faculté des sciences et de génie, Université Laval, Québec, QC G1V 0A6, Canada

' $\mathrm{CHU}$ de Québec, centre de recherche, Université Laval, Québec, QC G1L 3L5, Canada;

Index:

Section S1 - ATR sensing layer jig

Section S2 - Morphological measurements at ATR/PDMS interface

SectionS3 - Supplementary dynamic flow experiments

Section S4 - Assaying between water and DMF

Section S5 - A scan across a reaction device 


\section{Section S1 - ATR sensing layer jig}

Figure S1 shows the jig that was used to fabricate the ATR sensing layer.

(a)
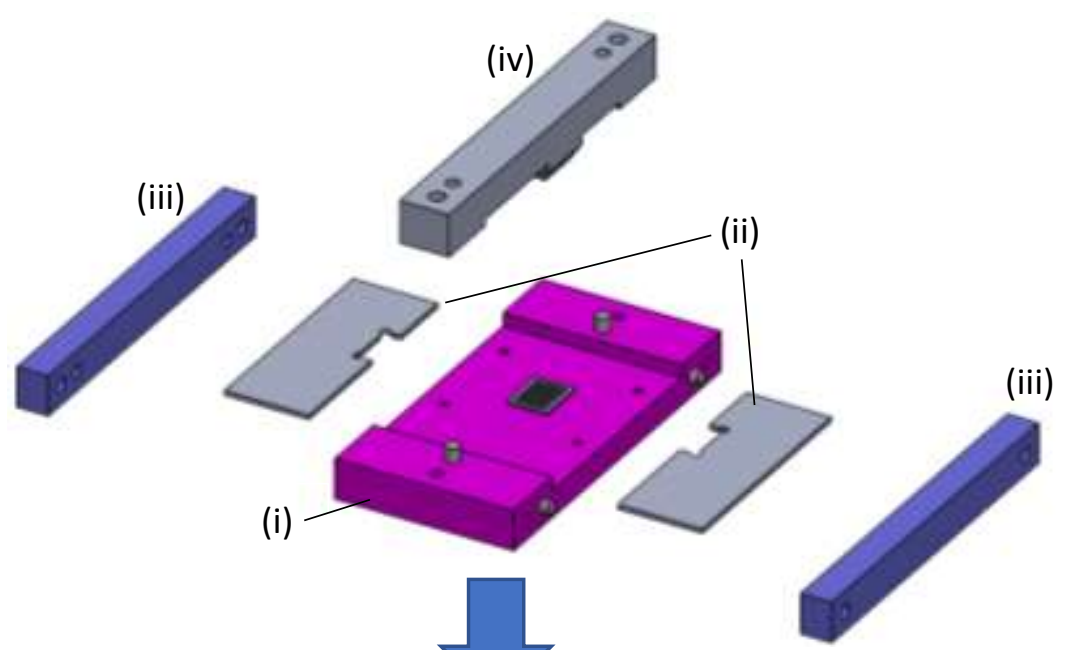

(b)

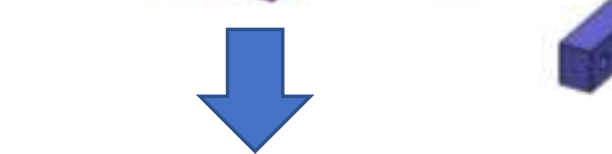

(iii)

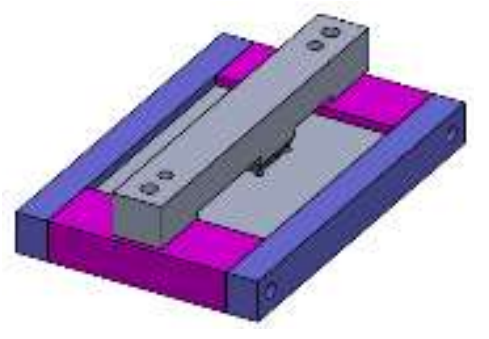

(c)
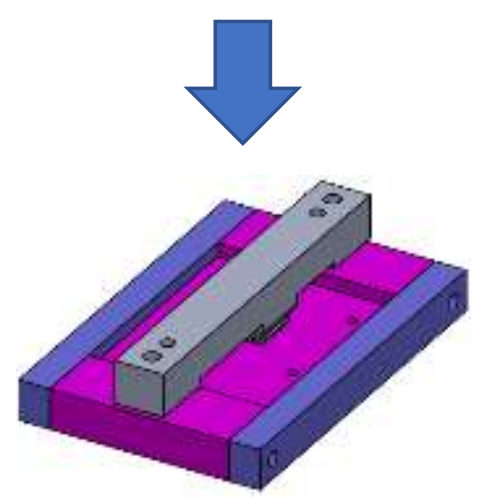

Figure S1. ATR alignment jig for fabrication of the ATR sensing layer. (a) Exploded view showing (i) jig base-plate (pink) with ATR crystal placed sensing side-down (grey), (ii) guiding plates, (iii) connecting sidebars and (iv) ATR immobilizing arm and Teflon plug with tapered edges. (b) Assembled view of jig with ATR guiding plates installed and (c) removed, in advance of pouring the PDMS. Glass-supported polyethylene thin film is not shown. 


\section{Section S2 - Morphological measurements at ATR/PDMS interface}

The morphology of junction part of crystal and PDMS was revealed by AFM measurement

(Figure S2). The height different between PDMS and crystal was less than $3 \mu \mathrm{m}$.

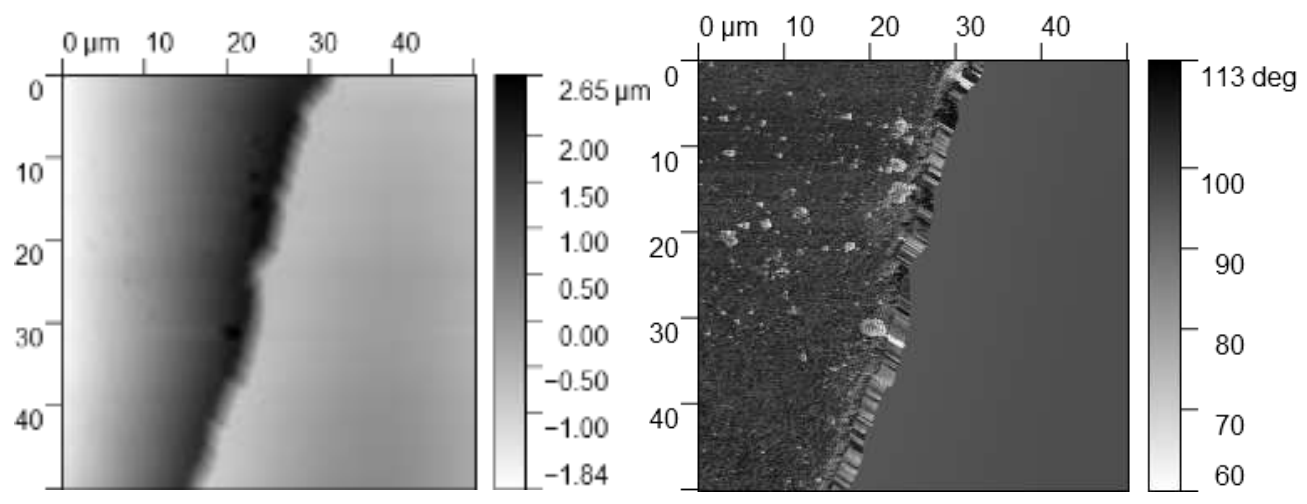

Figure S2. AFM images of crystal part at junction between crystal and PDMS in a) Height mode and b) phase mode. Left part is PDMS while right part is crystal.

\section{Section S3 - Supplementary dynamic flow experiments}

We demonstrated the generality of the technique shown in the main paper by oscillating between water and DMF. Both solvent spectra in Figure S3(a) are shown relative to an air background in order to show peaks for each solvent, as opposed to Figure $2 \mathrm{c}$ in the main paper in which water was used as a background. In Figure S3a, the water bands are visible, which enables a direct evaluation of any potential residual solvent from the previous flow pulse. Figure $\mathrm{S} 3 \mathrm{~b}$ shows the integrated band intensity as a function of time. Peak areas always returned to a zero value when flowing the other solvent, indicating that no residual solvent remained in the channel when the other was flowing. 
(a)

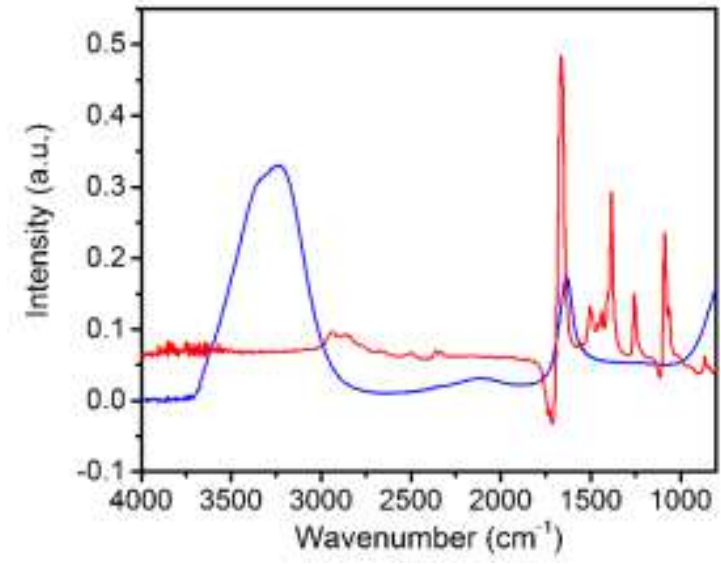

(b)

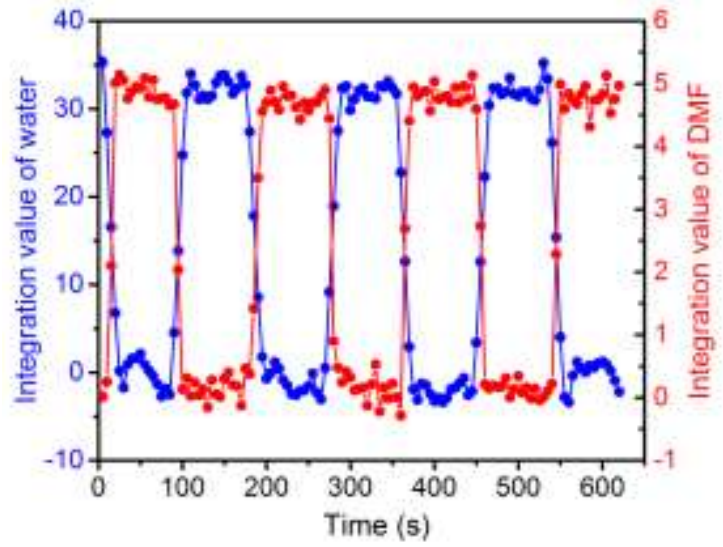

Figure S3. (a) Individual spectra of water (blue) and DMF (red) with unique peaks used for liquid identification being highlighted. (b) Result from alternating flow of water and DMF $\left(10 \mathrm{~mL} \cdot \mathrm{h}^{-1}\right)$.

\section{Section S4 - Assaying between water and DMF}

We used an air background obtained separately from each channel to observe the water and DMF spectra similar to section S3. This enabled a more direct route to validation of functionality of the device without cross - contamination. Figure S4 shows the individual spectra from each channel. No water bands at $3400 \mathrm{~cm}^{-1}$ are observed in the DMF spectra. Likewise, no DMFspecific bands (e.g. 1100 or $1400 \mathrm{~cm}^{-1}$ ) are observed in the water channels. This proves the measurement independence of each channel and expands the range of compatible solvents with the technique. 


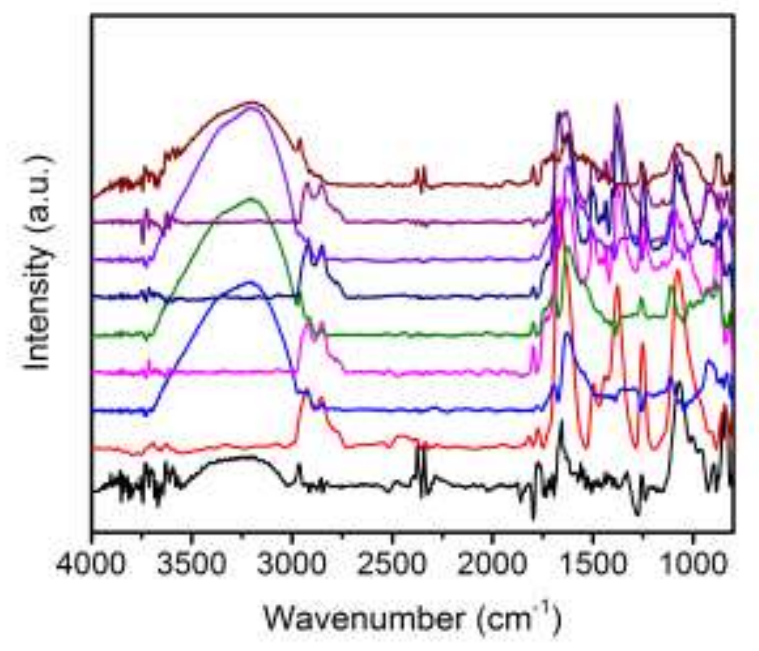

Figure S4. Flow water and DMF alternatively in 9 channel device and obtained spectra in each channel.

\section{Section S5 - A scan across a reaction device}
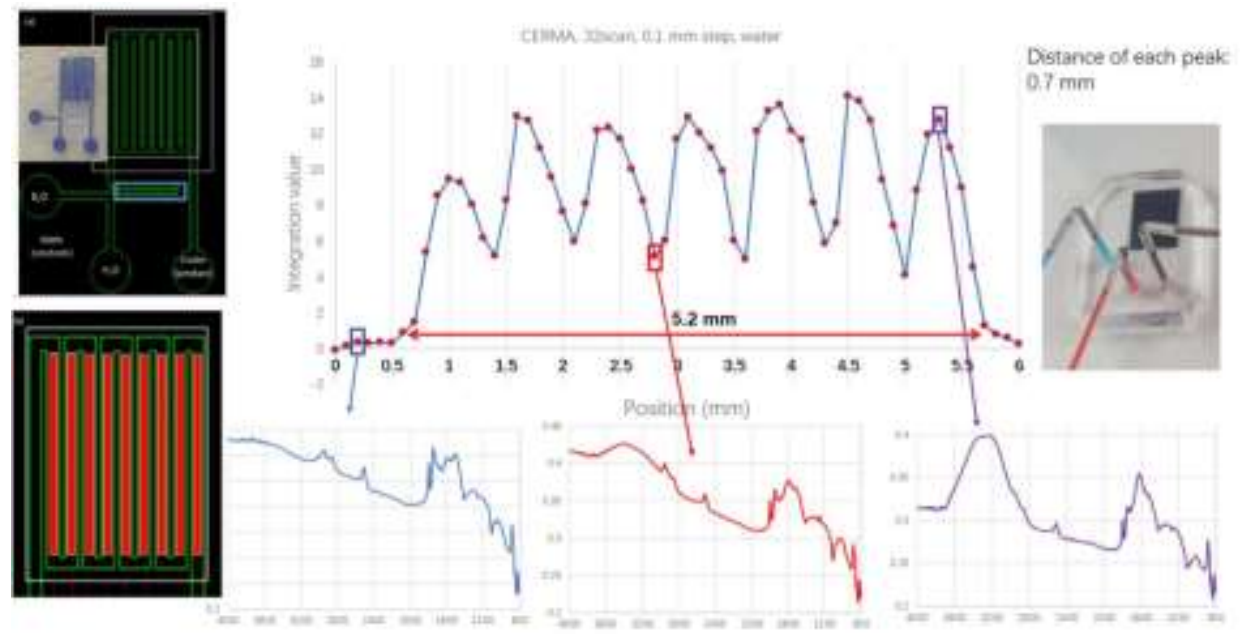

Figure S5. (a) CAD with device channel structure, and mixing channels highlighted (inset shows master mould). (b) zoom on reaction channel switchbacks with detection regtion in each 
channel (red boxes). (c) intensity of water band during an x-scan across the reactor device. Inset plots show spectra at select points. (d) image of the device with coulored liquid to show the contrast better. 